\title{
Leveraging Eye-gaze and Time-series Features to Predict User Interests and Build a Recommendation Model for Visual Analysis
}

\author{
Nelson Silva \\ Know-Center GmbH \\ TU Graz, CGV, Austria \\ nsilva@know-center.at \\ Vedran Sabol \\ Know-Center GmbH \\ Graz, Austria \\ vsabol@know-center.at
}

\author{
Tobias Schreck \\ TU Graz, Institute of Computer \\ Graphics and Knowledge \\ Visualisation (CGV), Austria \\ tobias.schreck@cgv.tugraz.at \\ Eva Eggeling \\ Fraunhofer Austria Research GmbH \\ Graz, Austria \\ eva.eggeling@vc.fraunhofer.at
}

\author{
Eduardo Veas \\ Know-Center $\mathrm{GmbH}$ \\ Graz, Austria \\ eveas@know-center.at
}

\begin{abstract}
We developed a new concept to improve the efficiency of visual analysis through visual recommendations. It uses a novel eye-gaze based recommendation model that aids users in identifying interesting time-series patterns. Our model combines time-series features and eye-gaze interests, captured via an eye-tracker. Mouse selections are also considered. The system provides an overlay visualization with recommended patterns, and an eye-history graph, that supports the users in the data exploration process. We conducted an experiment with 5 tasks where 30 participants explored sensor data of a wind turbine. This work presents results on pre-attentive features, and discusses the precision/recall of our model in comparison to final selections made by users. Our model helps users to efficiently identify interesting time-series patterns.
\end{abstract}

\section{CCS CONCEPTS}

- General and reference $\rightarrow$ Evaluation; • Human-centered computing $\rightarrow$ Visual analytics; $\bullet$ Information systems $\rightarrow R e c$ ommender systems;

\section{KEYWORDS}

Eye-tracking, model, recommend, evaluation, time-series, similarity, visual analytics

\section{ACM Reference Format:}

Nelson Silva, Tobias Schreck, Eduardo Veas, Vedran Sabol, Eva Eggeling, and Dieter W. Fellner. 2018. Leveraging Eye-gaze and Time-series Features to Predict User Interests and Build a Recommendation Model for Visual Analysis. In ETRA '18: 2018 Symposium on Eye Tracking Research and Applications, fune 14-17, 2018, Warsaw, Poland. ACM, New York, NY, USA, 9 pages. https://doi.org/10.1145/3204493.3204546

Permission to make digital or hard copies of part or all of this work for personal or classroom use is granted without fee provided that copies are not made or distributed for profit or commercial advantage and that copies bear this notice and the full citation on the first page. Copyrights for third-party components of this work must be honored.

For all other uses, contact the owner/author(s).

ETRA '18, fune 14-17, 2018, Warsaw, Poland

( 2018 Copyright held by the owner/author(s).

ACM ISBN 978-1-4503-5706-7/18/06.

https://doi.org/10.1145/3204493.3204546

\section{INTRODUCTION}

The analysis of the massive sensor data generated today, occupies a large part of specialists' time. Time-series data stored in large datasets requires analysis, which is not a straightforward process. Identifying interesting patterns and validating that they are under prescribed values, takes a significant part of the analysis. To assist, machine intelligent algorithms based on time-series similarity and specific retrieval, ranking strategies help recommend pattern candidates. These techniques are often insufficient, particularly, when considering changing user interests, which increases the user effort while performing comparisons between similar patterns. We contend that the task of visual analysis can be assisted with knowledge of gaze deployment.

Although Visual analysis is a human centered task, user interfaces are rather limited in terms of the amount of information they have about the current interests of users. User interests are either statically built into the knowledge base (pre-made profiles), or they are collect by the system, most often by tracing frequent users choices. We propose to adapt to the current user's interests and needs by continuously tracking them, in order to adapt the recommendations accordingly. Using sensor data to adapt the analytics process poses the challenge that the adaptive model needs to integrate distinct types of features, e.g., user's interests captured by the eye-tracker, and data features. In fact, inferring user's interests is by itself a complex task, as it encompasses selecting optimal sensing modalities, obtaining training data, learning models of user interests, and collecting appropriate sensing feedback. Eye-tracking technology can unveil rich information on a person's deployment of attention.

Our work aims at improving time-series similarity search applications by implementing techniques that can better support the users, and this is done in two ways. First, we recommend candidate patterns and guide the user attention via automated visualizations, controlled by a model that uses both eye-gaze and time-series features. Second, a visualization keeps track of eye-gaze interests (provenance visualization) and maintains the user's awareness of the analysis process. We seek to answer the following research 
questions: i) Does a model combining eye-gaze, time-series features, and user selections provides accurate recommendations? ii) Can it predict the final choices of the users? iii) Do users find the adaptive concept relevant for time-series analysis?

We target the prediction of multiple potentially relevant visual time-series patterns in respect to a given target pattern with a similar shape. We designed a study, where the user must assess similar time-series patterns. Our concept, provides on-the-fly support via visualizations that are automatically steered by the interests of the users, taking eye-gaze and data features as input. One of our gazecontrolled visualizations registers the eye-gaze interests history, i.e., eye-history graph. Another visualization, includes a new recommendation model, it is based on both eye-gaze and time-series features, it recommends the most potentially relevant candidate patterns, and overlays them on top of a representation of the target pattern, i.e., overlay visualization. In our study, we test that the users can be tracked using an eye-tracker, and we make sure that there are available 14 potentially relevant patterns to choose from.

\section{BACKGROUND}

To analyze user's interests, e.g., in market research, human-computer interaction, and visual analytics research, eye-tracking devices can be applied. Our concept combines both eye-gaze and time-series features to create a recommendation model. It aims to assist the users in visual search tasks, i.e., search for most similar time-series patterns in respect to a given target pattern. We support the users through visualization adaptation by automatically overlaying best potential pattern candidates. It combines visual attention analysis with interactive learning systems.

\subsection{Adaptive Interfaces and Users' Needs}

Many advances were made in the field of Intelligent and Adaptive User Interfaces (IAUIs), which demonstrate the advantages of IAUIs over traditional user interfaces, e.g., the ability of handling differences between preferences, or interests of several users. Despite the associated challenges, IAUIs have a wide range of applications, e.g., attention guidance in visual analytics. One can define Adaptive User Interfaces (AUIs) as applications that monitor the users' activity to identify usage patterns, and to automatically adjust interface components and content provided by the system, to changes in users' behaviors and interests [Alvarez-Cortes et al. 2007].

The processes of adaptation are complex and have a high impact on the users' acceptance of an interface. Visual attention strategies play an important role in adaptation [Bednarik 2012], [Göbel et al. 2016], allowing the study of visual and cognitive operations related to relevant or irrelevant information from cluttered scenes. It is possible to use visual attention to detect the right moment for adaptation and, as we propose, to prevent the information from changing when the user is looking at the visualizations. To learn more about the current interests and needs of the users, a system requires extra input methods. To this end, one can use a multiple sensors to track the user, either individually or through combination, i.e., sensor fusion. Sensor augmentation at the user workplace, is one important step to implement this sensor fusion. Typically, complex frameworks are in place to make sense of data coming from multiple sensors [Silva et al. 2014], [Eggeling et al. 2015].

\subsection{Eye-tracking and Visual Analytics}

In Human-Computer Interaction (HCI), eye movement tracking is commonly used to study usability issues [Jacob and Karn 2003]. Holmqvist et al. [Holmqvist et al. 2011] investigated multiple methods and measures for analyzing user attention patterns. Pool and Ball [Poole and Ball 2005] provided an introduction to the basics of eye-gaze movement. In our work, we follow an Area-of-Interest (AOI) metric approach under which, main areas of the application are considered individual AOIs and where each search result is also an AOI. Etemadpour et al. [Etemadpour et al. 2014] addresses the usage of eye-tracking in typical analysis tasks, like detecting clusters or correlations. Exhaustive descriptions of eye-tracking methods, including experimental design and setup, are offered by Duchowski [Duchowski 2003]. To make sense of eye-tracking data, extensive analysis and advanced visualization techniques are typically required [Blascheck et al. 2014], [Silva et al. 2016b]. Visualization techniques play an important role in evaluating user behavior, and in that regard several ideas were proposed by experts in eyetracking visualization [Blascheck et al. 2017].

An overview of visualization methods for analyzing eye data is provided by Andrienko et al. [Andrienko et al. 2012]. Moreover, Blascheck et al. [Blascheck et al. 2016] proposed a new approach to visualize eye-tracking data (eye-gaze sequences), which are important for the analysis of user behavior and overall system evaluation. Another interactive visual system for comparing eye-tracking data is discussed in Ristovski et al. [Ristovski et al. 2013]

AUIs facilitate the automatic adaptation of computer systems and are an important tool for data exploration tasks. In the field of visual analytics many applications can benefit from eye movement tracking. Eye-tracking is especially useful for the evaluation of visualizations [Kurzhals et al. 2014], as well as, for adapting the visual analytics process to current changes of users' behaviors and interests, e.g., control of user interfaces, exploration of large hierarchical structures and data provenance based on eye-tracking data [Silva et al. 2016a]. Another example is the exploration of large datasets through an automatic guided data exploration, by capturing user interests using an eye-tracker [Shao et al. 2017].

\subsection{Eye-gaze and Visual Attention}

A system that can track the user's activities and interests has great potential in terms of user support and guidance. Adaptation and support are usually achieved using recommender systems [Adomavicius and Tuzhilin 2005], which suggest potentially interesting items to the users. In our work, we infer users' interests by tracking their gaze movements using an affordable eye-tracker, i.e., EyeTribe (http://theeyetribe.com), which was used in previous research experiments [Stanislav et al. 2016].

Borji et al. [Borji et al. 2015] investigated the issue of inferring the search target in visual search by using eye-fixations. It was concluded that this inference is possible because during search, the users' attention and gaze are directed towards visual features that are similar to the ones of the search target. However, while the authors used realistic images of scenes with possibly interesting objects, in our work we focused on time-series patterns, i.e., plots. Abolhassani et al. [Haji-Abolhassani and Clark 2014] developed a probabilistic method based on the theory of Hidden Markov models 
(HMMs) to infer the visual-task of a viewer given the measured movement trajectories. Connor et all. [Connor et al. 2004] discusses the importance, advantages and disadvantages of top-down and bottom-up saliency-based attention models. Lu et al. [Lu et al. 2012] provided valuable insights into these two visual attention processes and presented a model to predict a visual attention map based on images. An experiment performed by Greene et al. [Greene et al. 2012], evaluated the use of eye scan-paths to predict the task of an observer. Important limitations in inferring complex cognitive processes from eye-movements were established. Prasov et al. [Prasov et al. 2007] investigated the role of eye-gaze to the automated identification of user attention. The results showed that gaze fixation intensity is crucial for attention prediction. Farnand et al, reported that image content has an inverse impact on gaze scanpath consistency: as the pictorial images become more complex, the observer attention becomes less focused, resulting in a reduced eye-gaze fixation consistency [Farnand et al. 2016].

To efficiently solve problems, users have to determine what information to look. They memorize this information for posterior comparison, but memory fades with time. The forgotten information may be compensated by using more seeking operations, or by adding visual properties (annotations, marks, and extra supportive information) [Conversy et al. 2010]. Our work takes advantage of this concept, by providing an eye-gaze overlay visualization which aims to support users in comparing pattern details.

\subsection{Time-series Patterns}

A time-series is a set of data on attribute values over time. Timeseries analysis allows the detection, or prediction of instances of a measure, based on past data. This allows the study of interesting events, i.e., detection of patterns leading to an engine malfunction. In time-series, a pattern is also seen as a time interval of interest. This time interval has specific behavior, and its visual representation is possible, e.g., in a plot. The task of finding similar patterns in respect to a certain target pattern of interest is not straightforward, and it requires specific analysis tools, due to the massive amount of data involved. Querying and exploring time-series patterns, is important in many fields, e.g., earthquake detection, autonomous driving. The goal of our work is to assist in these type of tasks, i.e., to support the process of search and comparison of time-series patterns. Our concept uses the Symbolic Aggregate approXimation (SAX) algorithm [Lin et al. 2003] for transforming time-series into strings. This speeds up the process of indexing and retrieval of timeseries patterns. A system that can automatically search and find the most relevant patterns must also use advanced similarity algorithms [Cassisi et al. 2012], such as, Constrained Dynamic Time Warping (CDTW) [Morel et al. 2018]. In this way, matching and retrieval of existent data patterns in respect to a given pre-annotated pattern, or event, can be accomplished.

\section{OVERVIEW OF OUR APPROACH}

Our concept offers support to the user in the visual search task of comparing similar shapes that leads to the identification and selection of interesting patterns. It comprises one overlay visualization, controlled by a recommendation model. This visualization supports the users by displaying potentially relevant patterns. The recommended patterns are overlaid on top of the target pattern to allow an easy comparison. In addition, the eye-history graph visualization, also controlled by eye-gaze data, keeps a history of eye-gaze visits of the user. This paper describes how our recommendation model combines eye-gaze features, time-series features, and pattern selections into one unified recommendation model.

\subsection{Concept Design}

Figure 1 shows our overall concept design. It is a web user interface based on an existing time-series platform which can index time-series signals, and allows the retrieval of similar patterns. It encodes, indexes and retrieves time-series signals, and it provides several well-known time-series similarity and ranking algorithms, e.g., DTW, CDTW. However, we limited our concept to only use the CDTW time-series similarity algorithm, as it consistently returns relevant pattern candidates [Morel et al. 2018]. Our concept displays the query pattern in the top left (1), this is relevant to the users during pattern comparison, because it shows all the details of the pattern. The search results area (3) displays the top 14 most relevant patterns found by the time-series similarity algorithm, i.e., CDTW. Note that the first search result returned is always an exact match to the query pattern of interest, the so called target pattern (2). The recommendation panel, displays an overlay visualization (4) with potentially interesting patterns. Every 2 seconds our model updates and if needed it displays the new relevant pattern recommendations. The recommendations depend on multiple user's interests, i.e., eye-gaze total time and total count, time-series features of items observed and selected. In Section 3.2 we further describe our recommendation model. A metadata table (5) with statistical information about a pattern and it is updated using mouse hover on each search result. Finally, an eye-history graph visualization (6) controlled by eye-gaze displays: IDs of patterns not yet explored (purple node); explored patterns IDs (green node), and the Top 3 most observed patterns IDs by considering the eye-gaze total time (blue node).

The concept has two modes of operation: test and task. The test mode verifies the eye-tracking components performance, highlighting in orange color each AOI according to the eye-gaze of the user. All main areas correspond to a specific eye-gaze AOI: extended signal area; each search result in the search results area; the overlay visualization; the metadata table; the eye-history graph. At a given time, only one area of interest is activated.

If the user continuously fixates one AOI, i.e, fixation time is higher than $200 \mathrm{~ms}$, the highlight of the AOI changes to green color ( $200 \mathrm{~ms}$ is the shortest amount of time to consider a valid fixation on a particular AOI). The recommendation model takes into account this newly activated area. When the user is able to activate each AOI the test mode is completed. The task mode is activated by clicking the "start task" button and the highlights become inactive. The application continues to track the eye-gaze of the user. In this mode the user executes a procedure described in Section 4.3.

\subsection{Model: Eye-gaze and Time-Series Features}

We created a new recommendation model concept based on eyegaze and time-series features. Figure 2 shows our concept. First, a set of time-series signals is indexed. Second, a time interval containing an interesting pattern for querying the indexed dataset is 


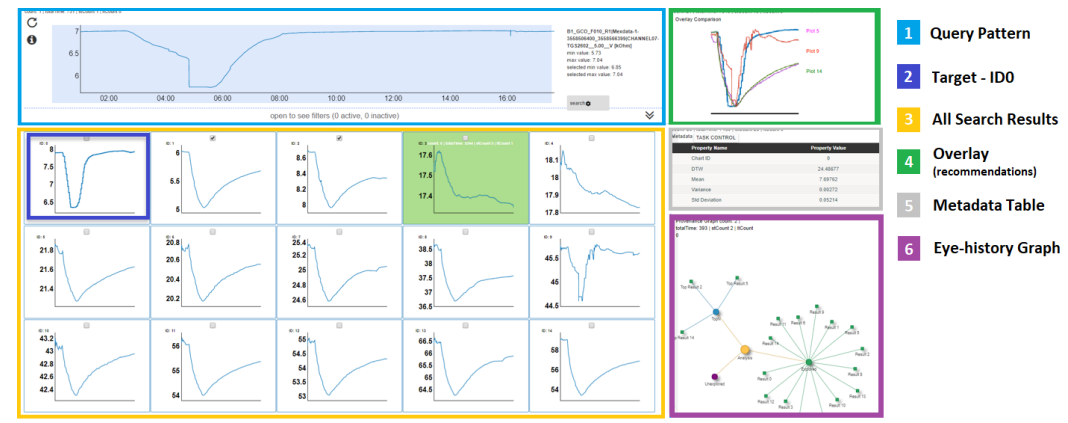

Figure 1: Gaze-assisted patterns' recommendation with all eye-tracked AOIs: retrieved patterns (yellow); Overlay visualization (green); Target-IDo (dark blue), identical to query pattern (light blue); history graph (violet); CDTW, mean, variance (gray).

pre-selected. Third, using a time-series similarity algorithm, the search results (time-series patterns similar in shape to the preselected time interval) are retrieved and displayed in the search results area. Fourth, as the user performs a task, an eye-tracker tracks and collects information about his/her eye-gaze behavior, i.e., AOI total fixation time, and AOI total fixation counts on each AOI fixated. Fifth, the user performs a task of visual inspection of interesting patterns, analyzes the recommendations given by our model, and selects the most interesting patterns. Sixth, the recommendation model updates both the overlay and the eye-history graph visualizations, i.e., eye-gaze and time-series features from the user's eye-gaze behavior and mouse selections. The overlay visualization assists the user in comparing the recommended patterns with the target. The eye-history graph visualization supports the user's awareness by allowing him/her to check which patterns have already been explored or not and, it also shows the top 3 most fixated patterns. The user can take advantage of this feature to recheck highly focused patterns, as these patterns might have caused more doubts to the user and therefore need an extra revision.

Figure 3 depicts the 3 steps required by the recommendation model to provide potentially relevant recommendations and the general formulas [Shimodaira 2014] used by our algorithm. In step 1 , the system extracts information about time-series features, i.e., CDTW, mean, variance, on every pattern observed by the user, and updates the Euclidean distances calculations between all patterns. Step 2 is similar to step 1 but for eye-gaze features, i.e., total fixation time and total fixation count. For each of these steps we generate a new similarity measure, i.e, a similarity measure for time-series features and a similarity measure for eye-gaze features. In step 3, the algorithm computes an Euclidean distance between every pattern, taking into account the new similarity measures for eyegaze and time-series features. These distances are updated every 2 seconds, and they consider changes in total fixation and total fixation count in each AOI, as well as, the time-series features of newly fixated or selected AOIs. Based on these new distances a new ranking score is applied, the search results are re-ranked and the top 3 most potentially relevant items are chosen. The 3 patterns with smaller distances to the target pattern are pushed to the top. We assume that the user observes the target pattern frequently, and that total fixation time, total fixation count, and time-series similarity score, play indeed an important role to determine the user interest on a certain pattern.

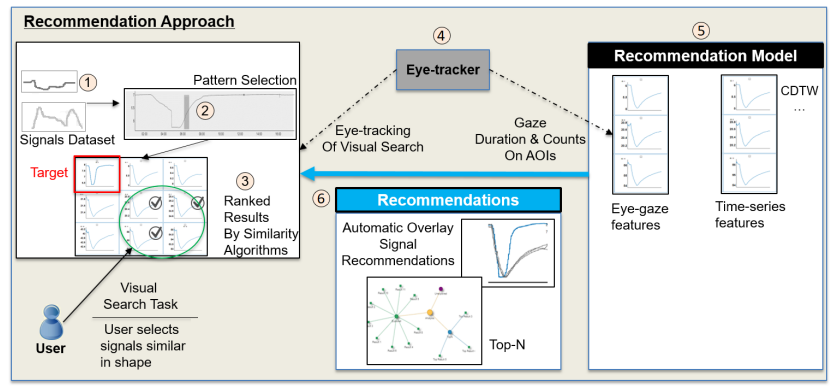

Figure 2: System Design: (1) time-series dataset with patterns; (2) query pattern; (3) ranked results; (4) eye-tracker; (5) recommendation model; (6) recommendations;

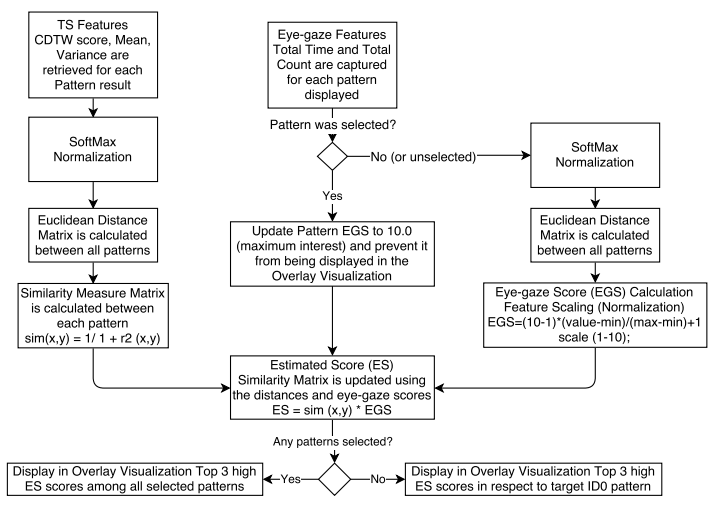

Figure 3: Recommendation model: it shows how to calculate the estimated final score to decide which patterns to overlay.

\section{METHOD USED IN OUR EXPERIMENT}

This experiment was conducted to validate our concept for recommending potentially interesting patterns. Our goal is to support the users when performing final selections by providing automatic visualizations steered by eye-gaze and time-series features.

\subsection{Participants}

We invited the university staff and students to take part in the experiment. Thirty two persons $(21 \mathrm{M}, 11 \mathrm{~F})$, whose ages varied between 22 and $50(\mathrm{M}=34.1, \mathrm{SD}=7.2)$ participated. Twenty two participants 
have normal vision, six wear glasses, and four wear contact lenses. None of the participants was subject to eye surgery. Our system was unable to collect reliable data for two of the participants, due to strong infrared protection coating in their glasses. We excluded them from the experimental results. Of 32 participants, 11 reported to have previous experience with time-series analysis.

\subsection{Apparatus}

In the experiment we used an affordable eye-tracker, i.e. EyeTribe, configured to capture data at a $30 \mathrm{fps}$ rate. One 15" G5 Studio HP Laptop (core i7) running Windows 10, and in addition a standard mouse was available. We placed the eye-tracker in the lower center of the laptop display, and the distance between the eye-tracker and the participant was $60 \mathrm{~cm}$. The experiment took place in a private and well illuminated room. The light conditions were kept stable. We had four applications running in the laptop, i.e. the server component for indexing and searching time-series patterns, one indexing Solr server, one Google chrome instance with 5 tabs (one per task) with our web front-end UI concept, one node.js application to capture and feed eye-tracking raw-data to our web front-end UI. The time-series dataset chosen for our experiment, was the "Wind Turbine - Gas sensor arrays in open sampling settings Data Set" from the UCI - Machine Learning Repository. We previously indexed this dataset in our time-series indexing and search system.

\subsection{Procedure}

At arrival, the participants received detailed written information about the experiment and explanations about our concept. The participants could ask questions that were answered by a single researcher. After signing a consent-form, the experiment began.

The experiment consisted of 5 similar tasks with 3 levels of increasing difficulty: 1 simple, 2 medium, and 2 hard. The levels were assigned by 5 time-series experts in our group. The task difficulty increased as the given pattern and retrieved patterns became more similar to each other, having only slight differences and making them more difficult to be compared visually. There was a time limit of 5 minutes for each task. The participants had to identify the total of four patterns similar in shape with respect to the given pattern target - ID0. Our concept displayed 14 different time-series patterns per task. To make sure that all participants had the same pattern results in each task, we selected and annotated five interesting time-series events from the dataset. Before starting a task the participants performed a 9 point eye-tracker initial calibration. Following the calibration, the researcher activated the test mode of the concept, and asked the participant to look to each AOI. To ensure an ideal eye-tracking throughout the experiment, we repeated the calibration process when necessary. The task started when the participants clicked the "task control" button, filled in the assigned participant id and task number, and clicked the "start task" button. The participants first check the target pattern - ID0, and acknowledge it by selecting it. The ID0 is always the exact match of the query pattern presented on top, and it is helpful for comparison with the other search results. This step also ensured that all participants started the task by looking at the ID0. After selecting ID0, the participants could explore the user interface freely. To complete a task, the participants searched and selected the four best pattern candidates, making use or not of the available recommendations. The participants performed the task at their own pace and finished the task by clicking the "stop task" button. After completing the five tasks, participants had to fill three questionnaires on-line: NASA-TLX (Perceived Workload Index) [Hart and Staveland 1988], SUS (System Usability Scale) [Brooke 1996], and a custom feedback questionnaire about specific functionalities of our application.

The experiment was comprised of 5 tasks of within-subjects design with levels of difficulty: 1 easy task (warm up), 2 medium and 2 hard tasks (difficult comparison of patterns, requiring more comparisons to the target and careful attention to detail). Our time-series experts group decided the difficulty level of each task. The more difficult the task, the harder it is to distinguish similar features among multiple patterns retrieved in the search results. We collected raw eye-gaze data from each participant, as well as other specific eyegaze data, including, AOIs total time and count, and scan-path. We recorded the mouse selections performed by the participant, and all recommendations generated by our model. After the experiment, we analyzed all participants answers per task. We computed an overall precision/recall, and F-score for the recommendation model. We took as ground truth, the analysis of the participants' pattern selections, by picking the top 4 most selected [Hernández-González et al. 2018]. Bellow we discuss the users' selections and show which patterns were most recommended per task, by our model.

\section{RESULTS}

We present preliminary results of our experiment with 30 participants. This section discusses results obtained from the analysis of the users' visual attention and eye-tracking data, our evaluation to the recommendation model and, the users' feedback on our concept.

\subsection{Visual Attention and Eye-tracking}

Figure 4 presents an attention map, which shows the participants' most fixated AOIs in Task1. These were, the target pattern (ID0), the overlay visualization (recommendations), the eye-history graph, and some specific pattern IDs. We analyzed the similarity between the fixations and sequences among our participants using the Levenshtein distance [Galgani et al. 2009]. For the fixation similarity the values varied between $50 \%$ and $96 \%$, meaning that the participants focused their attention on the same patterns. The sequences similarity analysis values varied between $6 \%$ and $16 \%$, indicating that the participants used different sequences strategies in their visual search for relevant patterns. While the Loci (fixation) similarity analysis between female and male participants is identical $(\sim 82 \%)$, there are differences in the sequence similarity $(\sim 17 \%)$.

Figure 5 demonstrates how we interactively investigated and compared the behavioral patterns of multiple participants at different time periods and on different scales. We investigated details of relevant pre-attentive features, visualization and combination of multiple logs, i.e., a correlation between AOIs eye-gaze data, recommendations given by the model, and final selections made by the participants. For example, we established that the participants spent $1 / 3$ of the time comparing the different patterns, and the remaining time on the selection process. Figure 6 shows an example of preattentive features analysis for the first $600 \mathrm{~ms}$ of a user's task. Note that our model recommends 3 plots (ID12, ID13 and ID14) and 


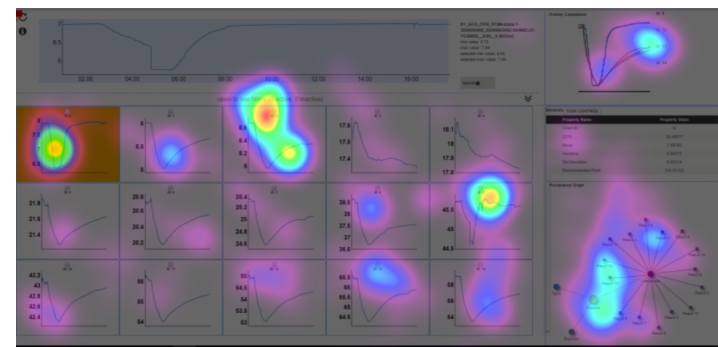

Figure 4: Attention Map: most fixated AOIs in Task1 (brighter areas). Created in OGAMA [Voßkühler et al. 2008]

simultaneously the user fixates the target pattern. After this period, the participant observes other not yet recommended patterns, while the recommender still shows the previous recommendations. Figure 7 presents what occurs before a participant selects an interesting pattern, i.e, ID9. Around time $51500 \mathrm{~ms}$, before making a selection at $55000 \mathrm{~ms}$, a recommendation for pattern ID9 is provided, and the participant really observes the ID9 recommendation in the overlay visualization (green bar). After this, the participant explores other patterns. Interestingly, before selecting ID9, the participant looks at the overlay visualization once more, at a time when the recommender does not display anymore the pattern ID9. Nevertheless, the participant still selects ID9, recommended $3500 \mathrm{~ms}$ before.

\subsection{Recommendation Model Evaluation}

The participants spent an average of 2.5 minutes (SD:1.23) on the 5 tasks. Table 1 shows the top 4 most selected patterns by participants in each task. We used these results as the ground truth in our preliminary analysis of the precision/recall scores of the recommendation model. As in many exploratory analytics tasks the ground truth, i.e., top 4 most selected patterns, depends on the users' perception. We split users in two groups: the ones who used the overlay visualization to make selections (12 users); and those who didn't (20 users). We compared the difference in responses to the ground truth using a T-test and established that there was no statistical difference between not using overlay $(\mathrm{M}=2.33, \mathrm{SD}=0.42)$ and using overlay $(\mathrm{M}=2.90, \mathrm{SD}=0.42)$ conditions, $\mathrm{t}(30)=2.04, \mathrm{p}=0.08$.

Table 1: Participants' top 4 final selections per task

\begin{tabular}{|c|c|c|c|c|c|c|c|c|c|}
\hline \multicolumn{2}{|c|}{ T1 } & \multicolumn{2}{|c|}{ T2 } & \multicolumn{2}{c|}{ T3 } & \multicolumn{2}{c|}{ T4 } & \multicolumn{2}{c|}{ T5 } \\
\hline ID & $\begin{array}{c}\text { Count } \\
(\#)\end{array}$ & ID & $\#$ & ID & $\#$ & ID & $\#$ & ID & $\#$ \\
\hline 13 & 26 & 2 & 31 & 13 & 26 & 1 & 32 & 13 & 32 \\
\hline 9 & 26 & 5 & 24 & 7 & 24 & 13 & 30 & 14 & 31 \\
\hline 14 & 20 & 10 & 21 & 8 & 19 & 7 & 30 & 3 & 31 \\
\hline 12 & 15 & 12 & 21 & 9 & 14 & 9 & 28 & 10 & 26 \\
\hline
\end{tabular}

Overall Results of Our Model - for the precision/recall analysis we assumed as the recommendation model would only recommend top 4 results during a task (results in Table 2).

Analysis of Total Fixation Time (FT) and Total Fixation Count (FC) - we analyzed the prediction of the participants' final selections by considering each one of the model components individually, i.e., total fixation time, total fixation count, and similarity
Table 2: Precision/Recall per task

\begin{tabular}{|l|c|c|c|c|c|}
\cline { 2 - 6 } \multicolumn{1}{c|}{} & \% T1 & $\% \mathrm{~T} 2$ & $\% \mathrm{~T} 3$ & $\% \mathrm{~T} 4$ & $\% \mathrm{~T} 5$ \\
\hline Accuracy & 85.7 & 31.3 & 41.5 & 26.3 & 42.7 \\
\hline Recall & 91.8 & 60.3 & 61.4 & 78.6 & 96.1 \\
\hline Precision & 88.9 & 18.6 & 16.9 & 21.5 & 40.2 \\
\hline F-score & 90.4 & 28.4 & 26.5 & 33.8 & 56.7 \\
\hline
\end{tabular}

Table 3: Total fixation time (FT) per AOI (all users and tasks)

\begin{tabular}{|l|c|c|c|c|c|c|}
\cline { 2 - 7 } \multicolumn{1}{c|}{ FT } & Signal & Overlay & Table & Graph & ID0 & Other \\
\hline MIN (s) & 0,017 & 18.250 & 0.017 & 0.017 & 0.017 & 2.3 \\
\hline AVG (s) & 6.7 & 14.7 & 5.6 & 3.7 & 6.8 & 7.1 \\
\hline MAX (s) & 36.8 & 92.4 & 42.4 & 19.3 & 14.0 & 28.6 \\
\hline SD & 0.1 & 0.3 & 0.1 & 0.1 & 0.1 & 5.0 \\
\hline
\end{tabular}

Table 4: Total fixation counts (FC) per AOI (all users, and tasks). Note: "Other" column = remaining AOIs.

\begin{tabular}{|l|c|c|c|c|c|c|}
\cline { 2 - 7 } \multicolumn{1}{c|}{ FC } & Signal & Overlay & Table & Graph & ID0 & Other \\
\hline MIN & 1 & 11 & 1 & 1 & 1 & 3 \\
\hline AVG & 29.1 & 46.6 & 18.0 & 15.6 & 21.4 & 28.6 \\
\hline MAX & 157 & 292 & 111 & 81 & 65 & 107 \\
\hline SD & 31.9 & 52.4 & 27.1 & 21.9 & 11.7 & 18.3 \\
\hline
\end{tabular}

Table 5: FT in top selections vs other patterns

\begin{tabular}{|l|c|c|c|c|c|}
\cline { 2 - 6 } \multicolumn{1}{c|}{} & S1 (13) & S2 (9) & S3 (14) & S4 (12) & Other \\
\hline MIN (s) & 0,1 & 0.2 & 0.6 & 0.3 & 2.3 \\
\hline AVG (s) & 4.6 & 2.8 & 3.6 & 2.8 & 7.1 \\
\hline MAX (s) & 10.8 & 14.4 & 13.5 & 11.7 & 28.6 \\
\hline SD & 3.4 & 3.4 & 3.1 & 2.8 & 5.0 \\
\hline
\end{tabular}

score. When using only FT it was possible to predict $27 \%$ of the selections in the correct order, and when using only FC it was possible to predict $7 \%$ of the selections in the correct order. If we do not consider the choice order, we can predict on average $24.2 \%$ (using only FC) and 35\% (FT) of selections. When using only the similarity score it was possible to predict $2 \%$ of the selections, independently of the choice order. Table 3 presents the total fixation time per AOI. The overlay area presents the most eye-gaze attention time (14.7s), demonstrating that participants observed the displayed recommendations while performing the tasks. Table 4 shows the total fixation counts per AOI, the overlay visualization received a higher number of visits. Table 5 shows the total fixation time for top selections of the users, while in Table 6, we show results considering the total fixation count. In general users spent more time and visited more AOIs that were not part of the top user selections.

\subsection{Feedback from Users}

NASA-TLX - Participants perceived a maximum workload score (overall task load) of 57.5. In summary, 10 participants perceived a workload score between 25 and 50, and 6 a workload score between 0 and $25.50 \%$ of participants reported low mental demand level and $50 \%$ a high mental demand level. We obtained similar results 


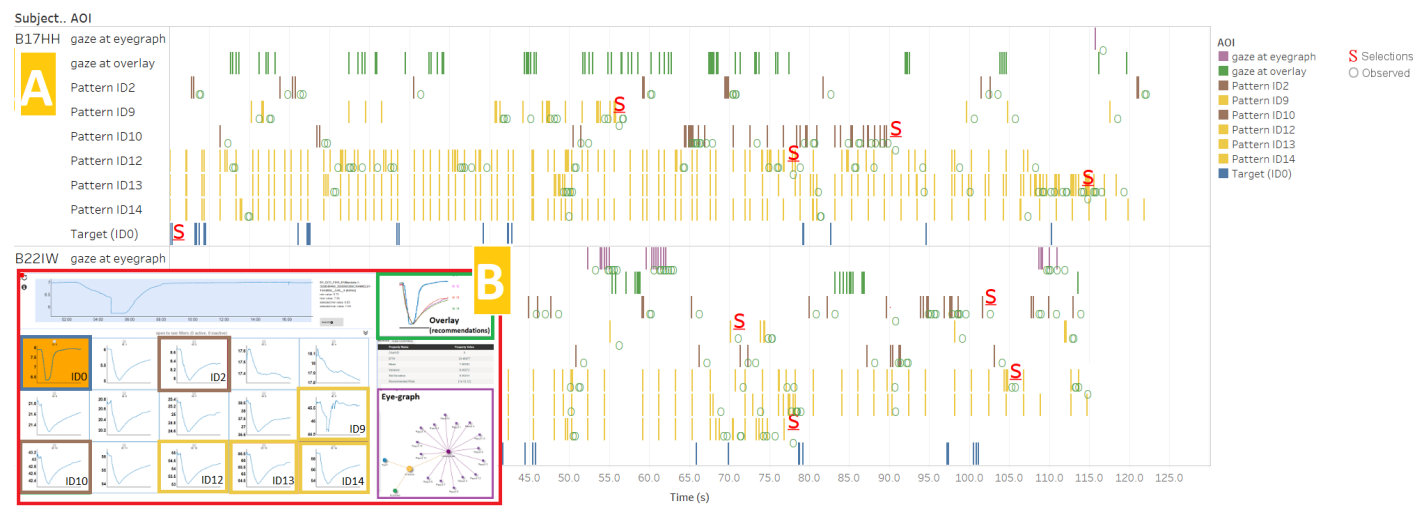

Figure 5: A) Patterns Exploration: with O - gaze at pattern; without O - recommended pattern; S - selection. B) Concept: Yellow - top 4 patterns; Brown - other patterns; Blue - target; Green - overlay; Purple - eye-graph.

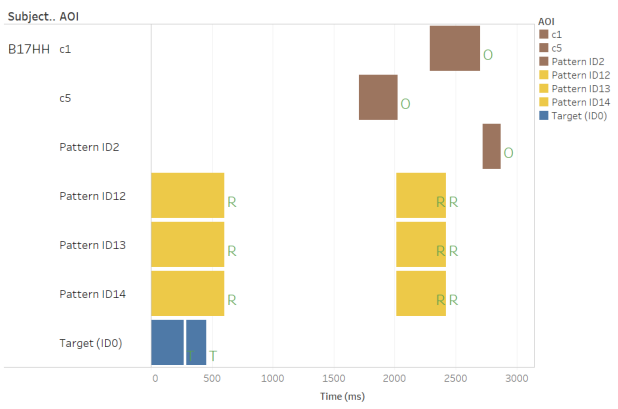

Figure 6: Pre-attentive Analysis: shows the participant behavior in the first $600 \mathrm{~ms}+$ of task 1 .

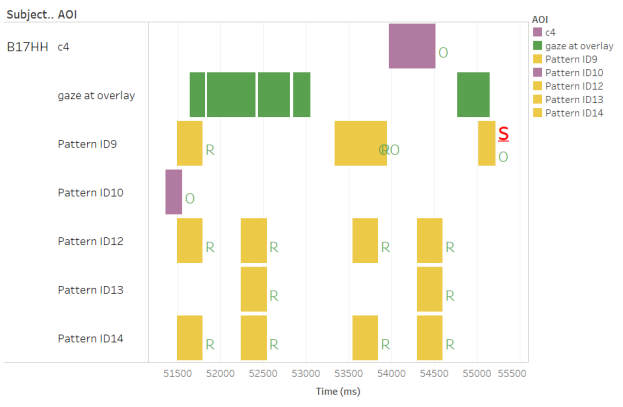

Figure 7: Analysis Before First Selection: it shows the participant behavior, $600 \mathrm{~ms}+$ before the first pattern selection.

Table 6: FC in top selections vs other patterns

\begin{tabular}{|l|c|c|c|c|c|}
\cline { 2 - 6 } \multicolumn{1}{c|}{} & S1 (13) & S2 (9) & S3 (14) & S4 (12) & Other \\
\hline MIN & 1 & 1 & 1 & 1 & 3 \\
\hline AVG & 19.4 & 13.0 & 16.1 & 11.5 & 28.6 \\
\hline MAX & 47 & 44 & 70 & 41 & 107 \\
\hline SD & 13.9 & 9.8 & 14.7 & 10.5 & 18.3 \\
\hline
\end{tabular}

for effort. Only 2 participants reported a high level of frustration, and all reported a low physical and a low temporal demand. 1 participant reported a low performance level.

SUS - this questionnaire targeted the usability evaluation of our concept. The score was 78.2 (grade B). Our concept only distances 2.1 points to score the A grade (80.3).

Custom feedback questionnaire - it included questions about our model recommendations, the overlay and eye-history graph visualizations, and the metadata table relevancy. Regarding the overlay visualization, $92 \%$ of the users liked it and made use of it. $68 \%$ reported to be quite satisfied with the patterns recommended by our recommendation model, which were overlaid while the user was performing each task. As for the metadata table, $76 \%$ of the users did not consider this information relevant for their task of identifying patterns similar in shape. Moreover, $20 \%$ of the users neither agreed or disagreed about the benefits of using this table for support, and only $4 \%$ stated that this information was sometimes relevant. For the eye-history graph, $40 \%$ reported that they neither agree or disagree with its relevancy for the tasks, while $32 \%$ do agree that it is relevant and $28 \%$ did not agree that it was helpful.

We asked about the participants' visual pattern search and comparison strategies. Surprisingly, $98 \%$ used a random strategy rather than methodically comparing each displayed pattern with the target pattern. $84 \%$ of users indicated that the overlay visualization helped to compare patterns with the target, $12 \%$ neither agree or disagree as to the usefulness of the overlaid plots for patterns comparison, and only $4 \%$ disagree with its relevancy. $70 \%$ reported to have used the recommended overlaid patterns to decide on pattern selections. $12 \%$ reported that they do not agree or disagree with having used the recommendations to make final decisions. Also, $17 \%$ of users reported that the recommendations had no influence in their final decision of selecting a pattern. We also asked the participants if they considered both similarities and dissimilarities between interesting patterns and the target. Here, $40 \%$ of the users reported that they looked for both similar and dissimilar patterns, and that the overlay visualization helped in both cases, i.e., to differentiate between interesting and irrelevant patterns for selection. $40 \%$ of users indicated that they were focused only in finding similar patterns, and $20 \%$ of users were unsure of their search and comparison strategy. 


\section{DISCUSSION}

We created a new concept to support users in the visual comparison and selection of most relevant patterns. Regarding the research questions (i, ii), the results are encouraging. There is evidence from NASA-TLX, SUS and a custom questionnaire, to support that our concept was well accepted by the participants in our study. They felt confident in selecting patterns while receiving automatic pattern recommendations from the system. It was stated that the automatic overlay allows to quickly establish if potential interesting patterns match the comparison target or not. According to the results, our model is able to suggest relevant patterns to the users. These patterns correspond to their current interests and provide timely support. The implications of achieving a good precision/recall when recommending potentially interesting patterns on-the-fly are important when creating more supportive and adaptive systems. The precision/recall analysis should be further investigated due to the adaptive nature of our recommendation model.

Our T-test results regarding the use of our concept with and without support of the overlay visualization suggest that, there is consensus on the user choices, regardless of the system used (with or without the overlay). As such, our system aids users in comparing and selecting the correct item, and it does not bias them towards arbitrary selections. We followed a strict approach for the evaluation, by comparing the top 4 selections of the users with the top 4 recommended patterns on each task. On tasks 1 and 5 users took more time to make their final selections, and the precision/recall results were acceptable. When the users immediately selected the relevant recommended patterns, the precision/recall results were worst since the model was prevented from recommending patterns that were already selected by the users. This penalizes a simple precision/recall analysis approach. We re-checked our data, and discovered that when the precision/recall was the lowest, i.e., in tasks 2, 3 and 4, this was because the users used the recommended patterns in early stages. In future, we want to evaluate recommendations given before a selection happens, and compare them with a random recommender algorithm, and use a Normalized Discounted Cumulative Gain (NDCG) evaluation approach [Wang et al. 2013].

Finally, in respect to research question (iii) the participants provided a positive feedback about our concept and the overall integration of visualizations. Regarding the eye-history graph visualization, the results did not confirm our expectation that the users wanted to know which patterns were already explored, or which patterns grabbed their attention the most. Although the metadata table with time-series statistical information and similarity score seemed important for some participants, its usage was very low in our experiment. Possibly, because our tasks did not require a strong numerical comparison. The participants liked the overlay visualization and they trusted the automatic recommendations. This is very encouraging for us to pursue new work and design new experiments that follow this automation and supportive path.

\subsection{Applications and Lessons Learnt}

Our work could be extended to other tasks, e.g., requiring guided attention or visual exploration, like comparison of photos and visual exploration of large datasets. We can consider the automatic redirection of user attention to details on visualizations or images, and the discovery of most important data features depending on the user's interests. These examples have application in other domains, e.g., reconstruction of historical artifacts, comparison of photos of missing persons, automatic information augmentation depending on interests and context, automated control of data exploration user interfaces. Considering the comparison of human skull images [Izbiki 2011] is a great example. Each skull image is converted to a time-series representation and then compared to other images using time-series similarity algorithms. Users could look to similar skull images (assuming a reference image) while our overlay visualization would display similar time-series corresponding to other interesting skull images. The user can then still look at familiar skull images, while our recommender model automatically overlays potentially similar time-series. Our concept can also be included in a visualization engine (as is our intention).

We learned that users are comfortable when interacting with our concept. However, they also presented critical comments and suggestions, e.g., simplifying the eye-history graph to display only unexplored patterns. In a multi-user scenario, a collaborative filtering recommender could be considered, which would require collecting a large set of user interests and votes. To take full advantage of this approach, users would have to select the same interval of time in a time-series, which is highly unlikely. Moreover, our concept already takes advantage of the continuous eye-tracking data feed to give recommendations.

\section{LIMITATIONS AND FUTURE WORK}

The limitations of our concept relate to the potential of using more eye-tracking metrics, e.g., eye scan-paths. While our approach is simple, it is able to assist the users with the proposed tasks. To this end, hidden Markov models could allow us to analyze interesting eye-gaze sequences and the ability to calculate interests probabilities, possibly contributing to better recommendation results. While adding one more visualization that updates automatically may draw attention resources from users, based on their feedback such attentional drifts are not considered distractions. Our visual search concept only considered 14 patterns per task. The same implementation can be used on a visual exploration system, which recurrently includes more search results based on the current interests of the users. The eye-history graph can be applied to pattern mining on the constructed graph in order to compare and learn from several users or for provenance analysis purposes.

\section{CONCLUSIONS}

Our approach is able to sense the users' interests, i.e., to infer potentially relevant patterns and use time-series features. As a result we created a recommender model that can support the users and automatically adapt multiple visualizations. This is highly relevant to the users and it is our main contribution. The feedback of the participants in our study was very reassuring for us to continue working on this topic, developing concepts to develop better adaptive visualization systems.

\section{ACKNOWLEDGMENTS}

This work is supported by TU Graz Open Access Publishing Fund. 


\section{REFERENCES}

Gediminas Adomavicius and Alexander Tuzhilin. 2005. Toward the Next Generation of Recommender Systems: A Survey of the State-of-the-Art and Possible Extensions. IEEE Trans. on Knowl. and Data Eng. 17, 6 (June 2005), 734-749.

V. Alvarez-Cortes, B. E. Zayas-Perez, V. H. Zarate-Silva, and J. A. Ramirez Uresti. 2007. Current Trends in Adaptive User Interfaces: Challenges and Applications. In Electronics, Robotics and Automotive Mechanics Conference (CERMA 2007). 312-317. https://doi.org/10.1109/CERMA.2007.4367705

Gennady Andrienko, Natalia Andrienko, Michael Burch, and Daniel Weiskopf. 2012 Visual Analytics Methodology for Eye Movement Studies. IEEE Transactions on Visualization and Computer Graphics 18, 12 (Dec. 2012), 2889-2898. https://doi.org/ 10.1109/TVCG.2012.276

Roman Bednarik. 2012. Expertise-dependent Visual Attention Strategies Develop over Time During Debugging with Multiple Code Representations. Int. F. Hum.-Comput. Stud. 70, 2 (Feb. 2012), 13. https://doi.org/10.1016/j.ijhcs.2011.09.003

T. Blascheck, M. John, K. Kurzhals, S. Koch, and T. Ertl. 2016. VA2: A Visual Analytics Approach for Evaluating Visual Analytics Applications. IEEE Transactions on Visualization and Computer Graphics 22, 1 (Jan 2016), 61-70. https://doi.org/10. 1109/TVCG.2015.2467871

T. Blascheck, K. Kurzhals, M. Raschke, M. Burch, D. Weiskopf, and T. Ertl. 2014. Stateof-the-Art of Visualization for Eye Tracking Data. In EuroVis - STARs, R. Borgo, R. Maciejewski, and I. Viola (Eds.). The Eurographics Association. https://doi.org/ 10.2312/eurovisstar.20141173

Tanja Blascheck, Kuno Kurzhals, Michael Raschke, Michael Burch, Daniel Weiskopf, and Thomas Ertl. 2017. Visualization of Eye Tracking Data: A Taxonomy and Survey. Computer Graphics Forum (2017).

Ali Borji, Andreas Lennartz, and Marc Pomplun. 2015. What do eyes reveal about the mind?: Algorithmic inference of search targets from fixations. Neurocomputing 149 (2015), 788 - 799. https://doi.org/10.1016/j.neucom.2014.07.055

John Brooke. 1996. SUS: A quick and dirty usability scale. (1996).

C. Cassisi, P. Montalto, M. Aliotta, A. Cannata, and A. Pulvirenti. 2012. Similarity Measures and Dimensionality Reduction Techniques for Time Series Data Mining. (2012). http://hdl.handle.net/2122/8082

Charles E. Connor, Howard E. Egeth, and Steven Yantis. 2004. Visual Attention: Bottom-Up Versus Top-Down. Current Biology 14, 19 (2004), R850 - R852.

Stéphane Conversy, Christophe Hurter, and Stéphane Chatty. 2010. A Descriptive Model of Visual Scanning. In Proceedings of the 3rd BELIV'10 Workshop: BEyond Time and Errors: Novel evaLuation Methods for Information Visualization (BELIV 10). ACM, New York, NY, USA, 35-42. https://doi.org/10.1145/2110192.2110198

Andrew T. Duchowski. 2003. Eye Tracking Methodology: Theory and Practice. SpringerVerlag New York, Inc., Secaucus, NJ, USA.

E. Eggeling, V. Settgast, N. Silva, M. Poiger, T. Zeh, and D. Fellner. 2015. The Sixth Sense of an Air Traffic Controller. In SID 2015. SesarJU. https://goo.gl/nDcrG8

R. Etemadpour, B. Olk, and L. Linsen. 2014. Eye-tracking investigation during visual analysis of projected multidimensional data with 2D scatterplots. In IVAPP 2014 233-246.

Susan Farnand, Preethi Vaidyanathan, and Jeff Pelz. 2016. Recurrence Metrics for Assessing Eye Movements in Perceptual Experiments. Fournal of Eye Movement Research 9, 4 (2016). https://bop.unibe.ch/index.php/JEMR/article/view/2539

F. Galgani, Y. Sun, P. L. Lanzi, and J. Leigh. 2009. Automatic analysis of eye tracking data for medical diagnosis. In 2009 IEEE Symposium on Computational Intelligence and Data Mining. 195-202. https://doi.org/10.1109/CIDM.2009.4938649

Fabian Göbel, Ioannis Giannopoulos, and Martin Raubal. 2016. The Importance of Visual Attention for Adaptive Interfaces. In Proceedings of the 18th International Conference on Human-Computer Interaction with Mobile Devices and Services Adjunct (MobileHCI '16). ACM, New York, NY, USA, 930-935.

Michelle R. Greene, Tommy Liu, and Jeremy M. Wolfe. 2012. Reconsidering Yarbus: A failure to predict observersâÁŹ task from eye movement patterns. Vision Research 62 (2012), 1 - 8. https://doi.org/10.1016/j.visres.2012.03.019

Amin Haji-Abolhassani and James J. Clark. 2014. An inverse Yarbus process: Predicting observers' task from eye movement patterns. Vision Research 103 (2014), 127 - 142 https://doi.org/10.1016/j.visres.2014.08.014

Sandra G Hart and Lowell E Staveland. 1988. Development of NASA-TLX (Task Load Index): Results of empirical and theoretical research. Human mental workload 1, 3 (1988), 139-183.

Jerónimo Hernández-González, Daniel Rodríguez, Iñaki Inza, Rachel Harrison, and José Antonio Lozano. 2018. Learning to classify software defects from crowds: A novel approach. Appl. Soft Comput. 62 (2018), 579-591.

Kenneth Holmqvist, Marcus Nystrom, Richard Andersson, Richard Dewhurst, Halszka Jarodzka, and Joost van de Weijer. 2011. Eye Tracking. A comprehensive guide to methods and measures. Oxford University Press. https://goo.gl/Gdaqsx

Mike Izbiki. 2011. Converting images into time series for data mining. (2011). https: //goo.gl/Q5ht4U Accessed: 2018-03-08.

R. J. K. Jacob and K. S. Karn. 2003. Eye Tracking in Human-Computer Interaction and Usability Research: Ready to Deliver the Promises. The Mind's eye: Cognitive The Mind's Eye: Cognitive and Applied Aspects of Eye Movement Research (2003), 573-603. http://www.sciencedirect.com.lt.ltag.bibl.liu.se/science
Kuno Kurzhals, Brian D. Fisher, Michael Burch, and Daniel Weiskopf. 2014. Evaluating visual analytics with eye tracking. In BELIV.

Jessica Lin, Eamonn Keogh, Stefano Lonardi, and Bill Chiu. 2003. A Symbolic Representation of Time Series, with Implications for Streaming Algorithms. In Proceedings of the 8th ACM SIGMOD Workshop on Research Issues in Data Mining and Knowledge Discovery (DMKD '03). ACM, New York, NY, USA, 2-11.

Y. Lu, W. Zhang, C. Jin, and X. Xue. 2012. Learning attention map from images. In 2012 IEEE Conference on Computer Vision and Pattern Recognition. 1067-1074.

Marion Morel, Catherine Achard, Richard Kulpa, and Séverine Dubuisson. 2018. Timeseries averaging using constrained dynamic time warping with tolerance. Pattern Recognition 74 (2018), 77-89. https://doi.org/10.1016/j.patcog.2017.08.015

Alex Poole and Linden J. Ball. 2005. Eye Tracking in HCI and Usability Research: Current Status and Future. In ProspectsâĂİ, Chapter in C. Ghaoui (Ed.): Encyclopedia of Human-Computer Interaction. Pennsylvania: Idea Group, Inc.

Zahar Prasov, Joyce Yue Chai, and Hogyeong Jeong. 2007. Eye Gaze for Attention Prediction in Multimodal Human-Machine Conversation. In Interaction Challenges for Intelligent Assistants, Papers from the 2007 AAAI Spring Symposium, 26-28 March 2007, Stanford University, CA, USA. 102-110.

G. Ristovski, M. Hunter, B. Olk, and L. Linsen. 2013. EyeC:Coordinated Views for Interactive Visual Exploration of Eye-Tracking Data. In International Conference on Information Visualisation. 239-248. https://doi.org/10.1109/IV.2013.32

Lin Shao, Nelson Silva, Eva Eggeling, and Tobias Schreck. 2017. Visual exploration of large scatter plot matrices by pattern recommendation based on eye tracking. In Proceedings of the 2017 ACM Workshop on Exploratory Search and Interactive Data Analytics. ACM, 9-16. https://doi.org/10.1145/3038462.3038463

Hiroshi Shimodaira. 2014. Similarity and recommender systems. (2014).

Nelson Silva, Volker Settgast, Eva Eggeling, Florian Grill, Theodor Zeh, and Dieter Fellner. 2014. Sixth sense-air traffic control prediction scenario augmented by sensors. In i-know 2014. ACM, 34. https://doi.org/10.1145/2637748.2638441

Nelson Silva, Lin Shao, Tobias Schreck, Eva Eggeling, and Dieter Fellner. 2016a. Visual Exploration of Hierarchical Data Using Degree-of-Interest Controlled by EyeTracking. In FMT 2016. https://goo.gl/Qp6k98

Nelson Silva, Lin Shao, T Schreck, Eva Eggeling, and Dieter W Fellner. 2016b. Sense.me - Open Source Framework for the Exploration and Visualization of Eye Tracking Data. In Proceedings of IEEE Conference on Information Visualization. Institute of Electrical and Electronics Engineers. https://goo.gl/NvnRZ8

Popelka Stanislav, Stachon Zdenek, Sasinka Cenek, and Dolezalova Jitka. 2016. EyeTribe Tracker Data Accuracy Evaluation and Its Interconnection with Hypothesis Software for Cartographic Purposes. (2016), 14 pages.

Adrian Voßkühler, Volkhard Nordmeier, Lars Kuchinke, and Arthur M. Jacobs. 2008. OGAMA (Open Gaze and Mouse Analyzer): Open-source software designed to analyze eye and mouse movements in slideshow study designs. Behavior Research Methods 40, 4 (01 Nov 2008), 1150-1162.

Yining Wang, Liwei Wang, Yuanzhi Li, Di He, Tie-Yan Liu, and Wei Chen. 2013. A Theoretical Analysis of NDCG Type Ranking Measures. CoRR (2013). 